\title{
Extremes of Order Statistics of Stationary Processes
}

\author{
Krzysztof Dębicki • Enkelejd Hashorva • \\ Lanpeng Ji · Chengxiu Ling
}

Received: date / Accepted: date

\begin{abstract}
Let $\left\{X_{i}(t), t \geq 0\right\}, 1 \leq i \leq n$ be independent copies of a stationary process $\{X(t), t \geq 0\}$. For given positive constants $u, T$, define the set of $r$ th conjunctions $C_{r, T, u}:=\left\{t \in[0, T]: X_{r: n}(t)>u\right\}$ with $X_{r: n}(t)$ the $r$ th largest order statistics of $X_{i}(t), t \geq 0,1 \leq i \leq n$. In numerous applications such as brain mapping and digital communication systems, of interest is the approximation of the probability that the set of conjunctions $C_{r, T, u}$ is not empty. Imposing the Albin's conditions on $X$, in this paper we obtain an exact asymptotic expansion of this probability as $u$ tends to infinity. Furthermore, we establish the tail asymptotics of the supremum of the order statistics processes of skew-Gaussian processes and a Gumbel limit theorem for the minimum order statistics of stationary Gaussian processes. As a by-product we derive a version of Li and Shao's normal comparison lemma for the minimum and the maximum of Gaussian random vectors.
\end{abstract}

Keywords Conjunction · Order statistics process · Albin's conditions · Generalized Albin constant · Skew-Gaussian process · Li and Shao's normal comparison lemma

Mathematics Subject Classification (2010) 60G10 - 60G70

Partial support from the Swiss National Science Foundation Project 200021-140633/1 and Marie Curie International Research Staff Exchange Scheme Fellowship within the 7th European Community Framework Programme (Grant No. RARE-318984) is kindly acknowledged. The first author also acknowledges partial support by Narodowe Centrum Nauki Grant No 2013/09/B/ST1/01778 (2014-2016).

K. Dȩbicki

Mathematical Institute, University of Wrocław,

pl. Grunwaldzki 2/4, 50-384 Wrocław, Poland

E. Hashorva · L. Ji · C. Ling

Department of Actuarial Science, University of Lausanne,

UNIL-Dorigny, 1015 Lausanne, Switzerland

E-mail: chengxiu.ling@unil.ch 


\section{Introduction and Main Result}

Let $\{X(t), t \geq 0\}$ be a stationary process with almost surely (a.s.) continuous sample paths and denote by $X_{1}, \ldots, X_{n}, n \in \mathbb{N}$ mutually independent copies of $X$. Of interest in this contribution is the $r$ th order statistics process $X_{r: n}$ of $X_{1}, \ldots, X_{n}$, i.e., for any $t \geq 0$

$$
X_{n: n}(t) \leq \cdots \leq X_{1: n}(t) .
$$

Throughout the paper, $X_{r: n}$ is referred to as the $r$ th order statistics process generated by the process $X$. Order statistics play a central role in many statistical applications. Naturally, the order statistics processes are of particular interest in statistical applications which involve the time-dynamics. If $X_{i}(t)$ is the value of a certain object (say image) $i$ measured at time point $t$, and $u$ is a fixed threshold, then the set of points that the $r$ th conjunction occurs before some time point $T$ is defined by

$$
C_{r, T, u}:=\left\{t \in[0, T]: X_{r: n}(t)>u\right\} .
$$

In applications it is of interest to calculate the probability that $C_{r, T, u}$ is not empty, which is given by

$$
p_{r, T}(u):=\mathbb{P}\left(C_{r, T, u} \neq \phi\right)=\mathbb{P}\left(\sup _{t \in[0, T]} X_{r: n}(t)>u\right) .
$$

Clearly, in an engineering context where $X_{i}$ 's model some random signals, $p_{r, T}(u)$ relates to the probability that at least $r$ signals overshoot the threshold $u$ at some point during the time interval $[0, T]$. Most prominent statistical applications, concerned with the analysis of the surface roughness during all machinery processes and functional magnetic resonance imaging (FMRI) data, relate to the calculations of $p_{r, T}(u)$. A methodology for the analysis of FMRI is established in the seminal contribution 31. Therein the authors derive approximations of $p_{n, T}(u)$ by calculating the expectation of Euler characteristic of $C_{n, T, u}$ for a fixed high threshold $u$.

For certain smooth Gaussian random fields approximations of $p_{n, T}(u)$ have been discussed in 7, 14,31, whereas results for some non-Gaussian random fields are derived in 9]. Exact asymptotic expansion of $p_{r, T}(u)$ for the class of stationary Gaussian processes $X$ was recently derived in [15]. Obviously, the Gaussian random field cannot be used to model phenomena and data sets that exhibit certain non-Gaussian characteristics such as skewness. It arises in many applied-oriented fields including engineering, medical science, agriculture and environmental studies; see, e.g., 1,8, 33. In recent years, new technologies such as FMRI and positron emission tomography have been used to collect data concerning the living human brain as well as astrophysics. As mentioned in the literature, these images can be efficiently modeled by stationary random fields.

Since the exact calculation of $p_{r, T}(u)$ is not possible in general, in this contribution we derive approximations of $p_{r, T}(u)$ for $u$ large. 
For the formulation of our main result we need to introduce Albin's conditions imposed on $X$ as suggested in $[2,3,6$. In what follows, let $\mathrm{D}$ be a non-empty subset of $\mathbb{R}$.

Condition A(D): (Gumbel MDA and conditional limit) Suppose that $X(0)$ has a continuous df with infinite right endpoint, and it is in the Gumbel maxdomain of attraction (MDA), i.e., for some positive scaling function $w(\cdot)$ we have as $u \rightarrow \infty$

$$
\mathbb{P}\left(X(0)>u+\frac{x}{w(u)}\right)=\mathbb{P}(X(0)>u) e^{-x}(1+o(1)), \quad \forall x \in \mathbb{R} .
$$

Let $q=q(u)$ satisfying $\lim _{u \uparrow \infty} q(u)=0$ be a strictly positive non-increasing function. Assume that for any $y \in \mathrm{D}$ there exists a random process $\left\{\xi_{y}(t), t \geq\right.$ $0\}$, such that for any grid of points $0<t_{1}<\cdots<t_{d}<\infty$ we have the convergence in distribution (denoted by $\stackrel{d}{\rightarrow}$ )

$$
\begin{aligned}
& \left(w(u)\left(X\left(q t_{1}\right)-u\right), \ldots, w(u)\left(X\left(q t_{d}\right)-u\right)\right) \mid(w(u)(X(0)-u)>y) \\
& \quad \stackrel{d}{\rightarrow}\left(\xi_{y}\left(t_{1}\right), \ldots, \xi_{y}\left(t_{d}\right)\right), \quad u \rightarrow \infty .
\end{aligned}
$$

Condition B: (Short-lasting-exceedance) For all positive constants $a, T$

$$
\lim _{N \rightarrow \infty} \limsup _{u \rightarrow \infty} \sum_{k=N}^{[T /(a q)]} \mathbb{P}(X(a q k)>u \mid X(0)>u)=0
$$

where $q$ is given as in condition $\mathrm{A}(\mathrm{D})$ and $[x]$ denotes the integer part of $x$.

Condition C: Suppose that there exist positive constants $\lambda_{0}, \rho, b, C$ and $d>1$ such that

$$
\mathbb{P}\left(X(q t)>u+\frac{\lambda}{w(u)}, X(0) \leq u \mid X(q t)>u\right) \leq C t^{d} \lambda^{-b}
$$

holds for all $u$ large and all $t$ positive such that $0<t^{\rho}<\lambda<\lambda_{0}$. Here $w$ and $q$ are given as in condition $\mathrm{A}(\mathrm{D})$.

Here we have chosen a simpler condition $\mathrm{C}$ than that in [2,3]. It has been shown in [6] that condition $\mathrm{C}$ above is sufficient for the validity of condition $\mathrm{C}$ given in 2,3; see also Proposition 2 in 4 .

Note that the Albin's conditions A(D),B,C given above are satisfied by many well-known stationary processes such as $\chi^{2}, \Gamma$ and $\sqrt{F}$ processes in 2. A concrete example is the Slepian process, see for other examples [6, 10. 29 30. However, showing the validity of these conditions requires in general significant efforts.

Let $\xi_{0}^{(i)}, i \leq n$ be independent copies of the random process $\xi_{0}$ given in condition $\mathrm{A}(\{0\})$. In order to derive the exact asymptotics of $p_{r, T}(u)$ we introduce the following constants

$$
\mathcal{A}_{r}:=\lim _{a \downarrow 0} \frac{1}{a} \mathbb{P}\left(\sup _{k \geq 1} \min _{1 \leq i \leq r} \xi_{0}^{(i)}(a k) \leq 0\right), \quad r \leq n,
$$


which we refer to as the generalized Albin constants. The finiteness and positiveness of it will be established in Theorem 1.1 below. For notational simplicity we set hereafter $c_{r, n}=n ! /(r !(n-r) !)$. Next, we state our principle result.

Theorem 1.1 Let $\left\{X_{r: n}(t), t \geq 0\right\}$ be the rth order statistics process generated by the stationary process $X$. If conditions $A(\{0\}), B$ and $C$ hold for $X$, then for any $T>0$, as $u \rightarrow \infty$,

$$
\mathbb{P}\left(\sup _{t \in[0, T]} X_{r: n}(t)>u\right)=T \mathcal{A}_{r} c_{r, n} \frac{(\mathbb{P}(X(0)>u))^{r}}{q(u)}(1+o(1)),
$$

where $\mathcal{A}_{r}$ defined in (1.7) is finite and positive.

This paper is organized as follows: In Section 2 we discuss an application of Theorem 1.1 concerning the skew-Gaussian processes and then derive the Gumbel limit theorem for the minimum order statistics process generated by a stationary Gaussian process $X$. All the proofs are presented in Section 3 Section 4 gives an Appendix which establishes a version of $\mathrm{Li}$ and Shao's normal comparison lemma for the minimum and maximum order statistics of Gaussian random vectors.

\section{Skew-Gaussian Processes and Gumbel Limit Theorem}

Throughout this section we assume that $\{X(t), t \geq 0\}$ is a centered stationary Gaussian process with a.s. continuous sample paths and covariance function $\rho(\cdot)$ such that, for some $\alpha \in(0,2]$

$$
\rho(t)<1, \quad \forall t>0 \text { and } \rho(t)=1-|t|^{\alpha}+o\left(|t|^{\alpha}\right), \quad t \rightarrow 0 .
$$

It is known (see, e.g., 3, 25, 17) that the process $X$ satisfies the assumptions of Theorem 1.1 with the process $\xi_{0}$ in condition $\mathrm{A}(\{0\})$ given by

$$
\xi_{0}(t)=\sqrt{2} Z(t)-t^{\alpha}+E, \quad t \geq 0,
$$

where $E$ is a unit exponential random variable (rv) and $\{Z(t), t \geq 0\}$ is a (independent of $E$ ) standard fractional Brownian motion (fBm) with Hurst index $\alpha / 2 \in(0,1]$, i.e., $Z$ is a centered Gaussian process with a.s. continuous sample paths and covariance function

$$
\operatorname{Cov}(Z(s), Z(t))=\frac{1}{2}\left(s^{\alpha}+t^{\alpha}-|s-t|^{\alpha}\right), \quad s, t \geq 0 .
$$

We note in passing that the findings of Theorem 1.1 for such $X$ coincide with those of Theorem 2.2 in [15. Our setup here is however more general than that of the aforementioned paper, as we demonstrate below. Let $\left\{X_{i}(t), t \geq\right.$ 
$0\}, i \leq m+1, m \in \mathbb{N}$ be independent copies of $X$. For any $\delta \in(0,1]$ define the skew-Gaussian process $\zeta$ as

$$
\zeta(t)=\delta|\boldsymbol{X}(t)|+\sqrt{1-\delta^{2}} X_{m+1}(t),|\boldsymbol{X}(t)|=\left(\sum_{i=1}^{m} X_{i}^{2}(t)\right)^{\frac{1}{2}}, \quad t \geq 0 .
$$

Theorem 2.2 Let $\left\{\zeta_{r: n}(t), t \geq 0\right\}$ be the rth order statistics process generated by the skew-Gaussian process $\zeta$. If the stationary Gaussian process $X$ has covariance function $\rho(\cdot)$ which satisfies (2.9), then for any $T>0$, as $u \rightarrow \infty$,

$$
\begin{aligned}
& \mathbb{P}\left(\sup _{t \in[0, T]} \zeta_{r: n}(t)>u\right) \\
& \quad=T \widetilde{\mathcal{A}}_{r, \alpha} c_{r, n} \delta^{r m-r} \frac{2^{r-r m / 2}}{(\Gamma(m / 2))^{r}} u^{\frac{2}{\alpha}+r m-2 r} e^{-\frac{r u^{2}}{2}}(1+o(1)),
\end{aligned}
$$

where $\widetilde{\mathcal{A}}_{r, \alpha}$ is determined by (1.7) with $\xi_{0}^{(i)}, i \leq n$ being $n$ independent copies of $\xi_{0}$ given in (2.10), and $\Gamma(\cdot)$ stands for the Euler Gamma function.

Remarks. a) The special case of Theorem 2.2 for $\delta=1$, coincides with that obtained in Corollary 7.3 in 25]; see also [17,20].

b) The Pickands constant $\mathcal{H}_{\alpha}$ coincides with $\widetilde{\mathcal{A}}_{1, \alpha}$ if $n=1$. It is well-known that $\mathcal{H}_{1}=1$ and $\mathcal{H}_{2}=1 / \sqrt{\pi}$. For other values of $\alpha$ the recent contribution [16 (see also the excellent monograph 32]) suggests an efficient algorithm to simulate $\mathcal{H}_{\alpha}$. For $n>1$ both calculation and simulation of $\widetilde{\mathcal{A}}_{r, \alpha}$ are open problems.

In extreme value analysis (see, e.g., [12,21,17]) it is also of interest to find some normalizing constants $a_{T}>0, b_{T} \in \mathbb{R}$ so that the linear normalization of the supremum $a_{T}\left(\sup _{t \in[0, T]} X_{r: n}(t)-b_{T}\right)$ converges in distribution as $T \rightarrow \infty$, where $X_{r: n}$ is the $r$ th order statistics process generated by the stationary Gaussian process $X$. The following theorem gives a Gumbel limit result for $X_{n: n}$ generated by a weakly dependent stationary Gaussian process.

Theorem 2.3 Let $\left\{X_{n: n}(t), t \geq 0\right\}$ be the minimum order statistics process generated by the stationary Gaussian process $X$ with covariance function $\rho(\cdot)$ satisfying (2.9). If $\rho(t) \ln t=o(1)$ holds as $t \rightarrow \infty$, then

$$
\lim _{T \rightarrow \infty} \sup _{x \in \mathbb{R}}\left|\mathbb{P}\left(a_{T}\left(\sup _{t \in[0, T]} X_{n: n}(t)-b_{T}\right) \leq x\right)-\exp \left(-e^{-x}\right)\right|=0
$$

where $\left(\right.$ set below $\left.D:=(n / 2)^{n / 2-1 / \alpha} \widetilde{\mathcal{A}}_{n, \alpha}(2 \pi)^{-n / 2}\right)$

$$
a_{T}=\sqrt{2 n \ln T}, b_{T}=\sqrt{\frac{2 \ln T}{n}}+\frac{\left(\frac{1}{\alpha}-\frac{n}{2}\right) \ln \ln T+\ln D}{\sqrt{2 n \ln T}} .
$$


Remarks. a) It follows from the proof of Theorem 2.3 that a similar result still holds for the maximum order statistics process $X_{1: n}$ under the same condition (since (4.35) holds for the maximum).

b) In several applications it is of interest to consider a random time interval $\mathcal{T}$ instead of $T$; see, e.g., [19,28. As in [28] our result in (2.13) can be extended for random intervals; we omit that result since it can be shown with similar arguments as in the aforementioned paper.

c) The deep contribution [26] shows that besides Gumbel limit theorems, of interest for applications is the growth of $\mathbb{E}\left(\left(\sup _{t \in[0, T]}\left|X_{n: n}(t)\right|\right)^{p}\right)$ for given $p>0$. In view of Theorem 2.2 (with $m=\delta=1$ ) and applying Lemma 4.5 in 27. we obtain

$$
\lim _{T \rightarrow \infty} \mathbb{E}\left(\left(\frac{\sup _{t \in[0, T]}\left|X_{n: n}(t)\right|}{\sqrt{2 / n} \ln T}\right)^{p}\right)=1 .
$$

\section{Proofs}

In this section, we present proofs of Theorems 1.1, 2.2 and 2.3. We shall rely on the methodology developed in the seminal paper [3]. As mentioned therein, checking the Albin's conditions for stationary processes is usually a hard task. In Section 3.1 we consider $X$ to be a stationary process with a.s. continuous sample paths. In Sections $3.2 \quad 3.3$ we concentrate on the special case where $X$ is a centered stationary Gaussian process with a.s. continuous sample paths and covariance function $\rho(\cdot)$ satisfying (2.9).

\subsection{Proof of Theorem 1.1}

We begin with some preliminary lemmas that will be used in the proof of Theorem 1.1] Unless otherwise specified, $\left\{X_{r: n}(t), t \geq 0\right\}$ denotes the $r$ th order statistics process generated by the stationary process $X$.

The next lemma plays a key role throughout the proofs. Since its proof is straightforward, we omit it. Recall that we defined $c_{r, n}=n ! /(r !(n-r) !)$.

Lemma 1 If $X(0)$ has a continuous distribution function, then for any $t \geq 0$

$$
\mathbb{P}\left(X_{r: n}(t)>u\right)=c_{r, n}(\mathbb{P}(X(t)>u))^{r}(1+o(1)), \quad u \rightarrow \infty .
$$

Lemma 2 If condition A(D) holds for $X$, then $X_{r: n}(0)$ has df in the Gumbel $M D A$ with scaling function $w_{r}(u)=r w(u)$. Further, for any grid of points $0<t_{1}<\cdots<t_{d}<\infty$ and all $y \in \mathrm{D}$ we have

$$
\begin{gathered}
\left(w_{r}(u)\left(X_{r: n}\left(q t_{1}\right)-u\right), \ldots, w_{r}(u)\left(X_{r: n}\left(q t_{d}\right)-u\right)\right) \mid\left(w_{r}(u)\left(X_{r: n}(0)-u\right)>r y\right) \\
\stackrel{d}{\rightarrow}\left(\min _{1 \leq i \leq r} r \xi_{y}^{(i)}\left(t_{1}\right), \ldots, \min _{1 \leq i \leq r} r \xi_{y}^{(i)}\left(t_{d}\right)\right), \quad u \rightarrow \infty,
\end{gathered}
$$

where $\xi_{y}^{(i)}, i \leq n$ are mutually independent copies of $\xi_{y}$ as in condition A(D). 
Proof. First, by (1.3) and (3.16)

$$
\mathbb{P}\left(X_{r: n}(0)>u+\frac{x}{r w(u)}\right)=\mathbb{P}\left(X_{r: n}(0)>u\right) e^{-x}(1+o(1)), \quad x \in \mathbb{R}
$$

meaning that $X_{r: n}(0)$ has df in the Gumbel MDA with $w_{r}(u)=r w(u)$. Further, it follows from (1.4) that the convergence in distribution

$$
\left(X_{i}^{*}\left(q t_{1}\right), \ldots, X_{i}^{*}\left(q t_{d}\right)\right) \mid\left(X_{i}^{*}(0)>r y\right) \stackrel{d}{\rightarrow}\left(r \xi_{y}^{(i)}\left(t_{1}\right), \ldots, r \xi_{y}^{(i)}\left(t_{d}\right)\right)
$$

holds as $u \rightarrow \infty$ for all $i \leq n, y \in \mathrm{D}$, where $X_{i}^{*}(t)=w_{r}(u)\left(X_{i}(t)-u\right)$. Let further $Y_{r}^{*}(t)=w_{r}(u)\left(X_{r: n}(t)-u\right)$ and fix a grid of points $0<t_{1}<\cdots<$ $t_{d}<\infty$. Next, we show that (3.17) holds when $r=n$. Indeed, for any given constants $y_{1}, \ldots, y_{d} \in \mathbb{R}$ by (3.18)

$$
\begin{aligned}
& \mathbb{P}\left(Y_{n}^{*}\left(q t_{1}\right)>y_{1}, \ldots, Y_{n}^{*}\left(q t_{d}\right)>y_{d} \mid Y_{n}^{*}(0)>n y\right) \\
& =\frac{\mathbb{P}\left(\min _{1 \leq i \leq n} X_{i}^{*}\left(q t_{j}\right)>y_{j}, 1 \leq j \leq d, \min _{1 \leq i \leq n} X_{i}^{*}(0)>n y\right)}{\mathbb{P}\left(\min _{1 \leq i \leq n} X_{i}^{*}(0)>n y\right)} \\
& \rightarrow \mathbb{P}\left(\min _{1 \leq i \leq n} n \xi_{y}^{(i)}\left(t_{1}\right)>y_{1}, \ldots, \min _{1 \leq i \leq n} n \xi_{y}^{(i)}\left(t_{d}\right)>y_{d}\right)
\end{aligned}
$$

as $u \rightarrow \infty$. Similarly, the claim of (3.17) holds for all $r<n$ if we show that, for any given constants $y_{1}, \ldots, y_{d} \in \mathbb{R}$

$$
\begin{aligned}
\mathbb{P} & \left(Y_{r}^{*}\left(q t_{1}\right)>y_{1}, \ldots, Y_{r}^{*}\left(q t_{d}\right)>y_{d} \mid Y_{r}^{*}(0)>r y\right) \\
= & \frac{\mathbb{P}\left(\min _{1 \leq i \leq r} X_{i}^{*}\left(q t_{j}\right)>y_{j}, 1 \leq j \leq d, \min _{1 \leq i \leq r} X_{i}^{*}(0)>r y\right)}{\mathbb{P}\left(\min _{1 \leq i \leq r} X_{i}^{*}(0)>r y\right)} \\
& \times\left(1+\Upsilon_{r}(u)\right), \text { with } \lim _{u \rightarrow \infty} \Upsilon_{r}(u)=0 .
\end{aligned}
$$

Next, we only present the proof for the case that $r=n-1$ and $d=1$; the other cases follow by similar arguments. By 3.16

$$
\mathbb{P}\left(Y_{n-1}^{*}(0)>(n-1) y\right)=n \mathbb{P}\left(\min _{1 \leq i \leq n-1} X_{i}^{*}(0)>(n-1) y\right)(1+o(1))
$$

as $u \rightarrow \infty$. Further

$$
\begin{aligned}
\mathbb{P}( & \left.Y_{n-1}^{*}\left(q t_{1}\right)>y_{1}, Y_{n-1}^{*}(0)>(n-1) y\right) \\
= & \mathbb{P}\left(Y_{n-1}^{*}\left(q t_{1}\right)>y_{1} \geq Y_{n}^{*}\left(q t_{1}\right), Y_{n}^{*}(0)>(n-1) y\right) \\
& +\mathbb{P}\left(Y_{n}^{*}\left(q t_{1}\right)>y_{1}, Y_{n-1}^{*}(0)>(n-1) y \geq Y_{n}^{*}(0)\right) \\
& +\mathbb{P}\left(Y_{n-1}^{*}\left(q t_{1}\right)>y_{1} \geq Y_{n}^{*}\left(q t_{1}\right), Y_{n-1}^{*}(0)>(n-1) y \geq Y_{n}^{*}(0)\right) \\
& +\mathbb{P}\left(Y_{n}^{*}\left(q t_{1}\right)>y_{1}, Y_{n}^{*}(0)>(n-1) y\right) \\
= & I_{1 u}+I_{2 u}+I_{3 u}+I_{4 u} .
\end{aligned}
$$


Since as $u \rightarrow \infty$

$$
\mathbb{P}\left(X_{n}^{*}\left(q t_{1}\right) \leq y_{1}, X_{n}^{*}(0)>(n-1) y\right) \leq \mathbb{P}\left(X_{n}(0)>u+\frac{y}{w(u)}\right)=o(1),
$$

we have

$$
I_{1 u}=n \mathbb{P}\left(\min _{1 \leq i \leq n-1} X_{i}^{*}\left(q t_{1}\right)>y_{1}, \min _{1 \leq i \leq n-1} X_{i}^{*}(0)>(n-1) y\right) o(1)
$$

and similarly $I_{2 u}=I_{1 u}(1+o(1))$. Using further the fact that

$$
\mathbb{P}\left(X_{n}^{*}\left(q t_{1}\right) \leq y_{1}, X_{n}^{*}(0) \leq(n-1) y\right)=1+o(1), u \rightarrow \infty,
$$

we have

$$
\begin{aligned}
I_{3 u}= & \sum_{i, i^{\prime} \leq n} \mathbb{P}\left(\min _{1 \leq j \leq n, j \neq i} X_{j}^{*}\left(q t_{1}\right)>y_{1}, X_{i}^{*}\left(q t_{1}\right) \leq y_{1},\right. \\
& \left.\quad \min _{1 \leq j^{\prime} \leq n, j^{\prime} \neq i^{\prime}} X_{j^{\prime}}^{*}(0)>(n-1) y, X_{i}^{*}(0) \leq(n-1) y\right) \\
= & n \mathbb{P}\left(\min _{1 \leq j \leq n-1} X_{j}^{*}\left(q t_{1}\right)>y_{1}, \min _{1 \leq j^{\prime} \leq n-1} X_{j^{\prime}}^{*}(0)>(n-1) y\right) \\
& \times \mathbb{P}\left(X_{n}^{*}\left(q t_{1}\right) \leq y_{1}, X_{n}^{*}(0) \leq(n-1) y\right) \\
& +c_{2, n} \mathbb{P}\left(\min _{1 \leq j \leq n-2} X_{j}^{*}\left(q t_{1}\right)>y_{1}, \min _{1 \leq j^{\prime} \leq n-2} X_{j^{\prime}}^{*}(0)>(n-1) y\right) \\
& \times \mathbb{P}\left(X_{n-1}^{*}\left(q t_{1}\right) \leq y_{1}, X_{n-1}^{*}(0)>(n-1) y\right) \mathbb{P}\left(X_{n}^{*}\left(q t_{1}\right)>y_{1}, X_{n}^{*}(0) \leq(n-1) y\right) \\
= & n \mathbb{P}\left(\min _{1 \leq j \leq n-1} X_{j}^{*}\left(q t_{1}\right)>y_{1}, \min _{1 \leq j^{\prime} \leq n-1} X_{j^{\prime}}^{*}(0)>(n-1) y\right)(1+o(1)) .
\end{aligned}
$$

Since in view of (3.19), for $k=0,1,2$, as $u \rightarrow \infty$,

$\mathbb{P}\left(\min _{1 \leq j \leq n-k} X_{j}^{*}\left(q t_{1}\right)>y_{1}, \min _{1 \leq j^{\prime} \leq n-k} X_{j^{\prime}}^{*}(0)>(n-1) y\right)=(\mathbb{P}(X(0)>u))^{n-k} O(1)$,

we conclude that $I_{4 u}=I_{3 u} O(1)$, and further that (3.20) holds for $r=n-1$ and $d=1$. This completes the proof.

Lemma 3 If condition $B$ is satisfied by $X$, then for any a, $T$ positive

$$
\lim _{N \rightarrow \infty} \limsup _{u \rightarrow \infty} \sum_{k=N}^{[T /(a q)]} \mathbb{P}\left(X_{r: n}(a q k)>u \mid X_{r: n}(0)>u\right)=0
$$

Proof. First, since for all integers $k \geq 1$ and any $u$ positive

$$
\begin{gathered}
\mathbb{P}\left(X_{n: n}(a q k)>u \mid X_{n: n}(0)>u\right)=\frac{\mathbb{P}\left(X_{n: n}(a q k)>u, X_{n: n}(0)>u\right)}{\mathbb{P}\left(X_{n: n}(0)>u\right)} \\
=(\mathbb{P}(X(a q k)>u \mid X(0)>u))^{n} \leq \mathbb{P}(X(a q k)>u \mid X(0)>u)
\end{gathered}
$$


holds, condition B implies the claim for $r=n$. If $r<n$, then with similar arguments as in (3.20) we have for large $u$

$$
\begin{aligned}
\mathbb{P}\left(X_{r: n}(a q k)>u \mid X_{r: n}(0)>u\right) & =(\mathbb{P}(X(a q k)>u \mid X(0)>u))^{r}\left(1+\Upsilon_{r}(u)\right) \\
& \leq K(\mathbb{P}(X(a q k)>u \mid X(0)>u))^{r}
\end{aligned}
$$

holds for some $K>0$, hence again condition B establishes the proof.

Lemma 4 If condition $C$ is satisfied by $X$ with the parameters as therein, then there exists some positive constant $C^{*}$ such that for all $u$ large

$$
\mathbb{P}\left(X_{r: n}(q t)>u+\frac{\lambda}{w(u)}, X_{r: n}(0) \leq u \mid X_{r: n}(q t)>u\right) \leq C^{*} t^{d} \lambda^{-b}
$$

holds for any $t$ positive satisfying $0<t^{\rho}<\lambda<\lambda_{0}$.

Proof. By condition $\mathrm{C}$, for sufficiently large $u$ and $C^{*}=n C, r=n$

$$
\begin{aligned}
& \mathbb{P}\left(X_{n: n}(q t)>u+\frac{\lambda}{w(u)}, X_{n: n}(0) \leq u \mid X_{n: n}(q t)>u\right) \\
& \leq \sum_{i=1}^{n} \frac{\mathbb{P}\left(X_{n: n}(q t)>u+\frac{\lambda}{w(u)}, X_{i}(q t) \leq u\right)}{\mathbb{P}\left(X_{n: n}(q t)>u\right)} \\
& \leq \sum_{i=1}^{n} \frac{\mathbb{P}\left(X_{i}(q t)>u+\frac{\lambda}{w(u)}, X_{i}(0) \leq u, \min _{1 \leq j \leq n, j \neq i} X_{j}(q t)>u\right)}{(\mathbb{P}(X(q t)>u))^{n}} \\
& =\sum_{i=1}^{n} \frac{\mathbb{P}\left(X_{i}(q t)>u+\frac{\lambda}{w(u)}, X_{i}(0) \leq u\right)}{\mathbb{P}(X(q t)>u)} \\
& =\sum_{i=1}^{n} \mathbb{P}\left(X_{i}(q t)>u+\frac{\lambda}{w(u)}, X_{i}(0) \leq u \mid X_{i}(q t)>u\right) \\
& \leq C^{*} t^{d} \lambda^{-b}
\end{aligned}
$$

holds for all $t$ positive satisfying $0<t^{\rho}<\lambda<\lambda_{0}$. If $r<n$, then with similar arguments as in (3.20)

$$
\begin{aligned}
& \mathbb{P}\left(X_{r: n}(q t)>u+\frac{\lambda}{w(u)}, X_{r: n}(0) \leq u\right) \\
& \quad=c_{r, n} \mathbb{P}\left(\min _{1 \leq i \leq r} X_{i}(q t)>u+\frac{\lambda}{w(u)}, \min _{1 \leq i \leq r} X_{i}(0) \leq u\right)(1+o(1))
\end{aligned}
$$

holds as $u \rightarrow \infty$. Consequently, it follows from (3.23) that there exists some positive constant $C^{*}$ such that

$$
\mathbb{P}\left(X_{r: n}(q t)>u+\frac{\lambda}{w(u)}, X_{r: n}(0) \leq u \mid X_{r: n}(q t)>u\right) \leq C^{*} t^{d} \lambda^{-b}
$$


holds for all $t>0$ and $0<t^{\rho}<\lambda<\lambda_{0}$ establishing thus the proof.

Proof of Theorem 1.1. The proof is based on an application of Theorem 1 in [3], see also Lemma A in [6]. It follows from Lemmas 2, 3] and [4 that the conditions of Theorem 1 in 3 are satisfied, hence for any $T>0$

$$
\mathbb{P}\left(\sup _{t \in[0, T]} X_{r: n}(t)>u\right)=T \mathcal{A}_{r} \frac{\mathbb{P}\left(X_{r: n}(0)>u\right)}{q(u)}(1+o(1)), \quad u \rightarrow \infty,
$$

where the limit in the right-hand side of (1.7) exists with $\mathcal{A}_{r} \in(0, \infty)$. Hence the proof follows from (3.16).

\subsection{Proof of Theorem 2.2}

In the following, we focus on the special case where the process $X$ is a centered stationary Gaussian process with a.s. continuous sample paths and covariance function $\rho(\cdot)$ satisfying (2.9) . Before proceeding to the proof of Theorem 2.2 . we present four lemmas.

Lemma 5 If $\{\zeta(t), t \geq 0\}$ is given as in (2.11), then

$$
\mathbb{P}(\zeta(0)>u)=\delta^{m-1} \frac{2^{1-m / 2}}{\Gamma(m / 2)} u^{m-2} \exp \left(-\frac{u^{2}}{2}\right)(1+o(1))
$$

and further the convergence in probability

$$
|\boldsymbol{X}(0)| \mid(\zeta(0)>u) \stackrel{p}{\rightarrow} \infty
$$

holds as $u \rightarrow \infty$.

Proof. Since $|\boldsymbol{X}(0)|^{2} / 2$ has $\operatorname{Gamma}(m / 2,1)$ distribution, we have

$$
\mathbb{P}(|\boldsymbol{X}(0)|>u)=\frac{2^{1-m / 2}}{\Gamma(m / 2)} u^{m-2} \exp \left(-\frac{u^{2}}{2}\right)(1+o(1)), \quad u \rightarrow \infty .
$$

Hence, Theorem 2.2 in [18] implies for any $\delta \in(0,1]$

$$
\mathbb{P}(\zeta(0)>u)=\delta^{m-1} \mathbb{P}(|\boldsymbol{X}(0)|>u)(1+o(1)), \quad u \rightarrow \infty .
$$

Clearly, (3.24) holds for $\delta=1$. Next, taking a constant $c$ such that $1<c<$ $1 / \sqrt{1-\delta^{2}}$ for $\delta \in(0,1)$, it follows from the proof of Lemma 2.3 in [18, that

$$
\lim _{u \rightarrow \infty} \mathbb{P}\left(\left(1-c \sqrt{1-\delta^{2}}\right) u<\delta|\boldsymbol{X}(0)|<c \delta u \mid \zeta(0)>u\right)=1,
$$

implying thus (3.24). Hence the proof is complete. 
Lemma 6 Let $\{\zeta(t), t \geq 0\}$ be given as in 2.11). If the covariance function $\rho(\cdot)$ of the generic stationary Gaussian process $X$ satisfies (2.9), then for any grid of points $0<t_{1}<\cdots<t_{d}<\infty$ the joint convergence in distribution

$$
\left(u\left(\zeta\left(q t_{1}\right)-u\right), \ldots, u\left(\zeta\left(q t_{d}\right)-u\right)\right) \mid(\zeta(0)>u) \stackrel{d}{\rightarrow}\left(\xi_{0}\left(t_{1}\right), \ldots, \xi_{0}\left(t_{d}\right)\right)
$$

holds as $u \rightarrow \infty$, where the process $\xi_{0}$ is given by (2.10) and $q=q(u):=$ $u^{-2 / \alpha}$

Proof. By Lemma 5, for any $s \in \mathbb{R}$

$$
\lim _{u \rightarrow \infty} \frac{\mathbb{P}\left(\zeta(0)>u+\frac{s}{u}\right)}{\mathbb{P}(\zeta(0)>u)}=e^{-s} .
$$

Consequently, we have the convergence in distribution

$$
u(\zeta(0)-u) \mid(\zeta(0)-u>0) \stackrel{d}{\rightarrow} E, \quad u \rightarrow \infty,
$$

with $E$ a unit exponential rv. In view of Theorem 5.1 in [11, it suffices to show that as $u \rightarrow \infty$

$$
\begin{aligned}
& \left(u\left(\zeta\left(q t_{1}\right)-u\right), \ldots, u\left(\zeta\left(q t_{d}\right)-u\right)\right) \mid\left(\zeta(0)=u_{x}\right) \\
& \quad \stackrel{d}{\rightarrow}\left(\sqrt{2} Z\left(t_{1}\right)-t_{1}^{\alpha}+x, \ldots, \sqrt{2} Z\left(t_{d}\right)-t_{d}^{\alpha}+x\right), \quad u_{x}:=u+x / u
\end{aligned}
$$

holds for all $d \geq 1$ and almost all $x>0$ with $Z$ a standard fBm with Hurst index $\alpha / 2$. Define

$$
X_{i}^{*}=\rho\left(q t_{j}\right) X_{i}(0), \quad \Delta_{i}\left(t_{j}\right)=X_{i}\left(q t_{j}\right)-X_{i}^{*}, \quad i \leq m+1, j \leq d .
$$

For any $u>0$ and $j \leq d$, we have

$$
\begin{aligned}
& \left(u\left[\zeta\left(q t_{j}\right)-u\right]-x\right) \mid\left(\zeta(0)=u_{x}\right) \\
& =\left(\delta u\left(\sqrt{\sum_{i=1}^{m}\left(X_{i}^{2}(0)+2 X_{i}^{*} \Delta_{i}\left(t_{j}\right)-\left(1-\rho^{2}\left(q t_{j}\right)\right) X_{i}^{2}(0)+\Delta_{i}^{2}\left(t_{j}\right)\right)}-|\boldsymbol{X}(0)|\right)\right. \\
& \left.\quad+\sqrt{1-\delta^{2}} u\left(\Delta_{m+1}\left(t_{j}\right)-\left(1-\rho\left(q t_{j}\right)\right) X_{m+1}(0)\right)\right) \mid\left(\zeta(0)=u_{x}\right) \\
& =: \delta A_{u}+\sqrt{1-\delta^{2}} B_{u} .
\end{aligned}
$$

Let $Z_{i}, i \leq m+1$ be mutually independent copies of $Z$. In view of (2.9) for $s, t>0$ and $i \leq m+1$

$$
\lim _{u \rightarrow \infty} u^{2} \operatorname{Cov}\left(\Delta_{i}(s), \Delta_{i}(t)\right)=s^{\alpha}+t^{\alpha}-|s-t|^{\alpha}=2 \operatorname{Cov}\left(Z_{i}(s), Z_{i}(t)\right),
$$

which implies the following convergence of finite-dimensional distributions

$$
\left\{u \Delta_{i}(t), t \geq 0\right\} \stackrel{d}{\rightarrow}\left\{\sqrt{2} Z_{i}(t), t \geq 0\right\}, \quad u \rightarrow \infty, \quad i \leq m+1 .
$$


By the independence of $\Delta_{i}$ 's and $X_{i}$ 's, the $Z_{i}$ 's can be chosen to be independent of $\zeta(0)$. Further, since $\left(X_{1}(0), \ldots, X_{m+1}(0)\right)$ is a centered Gaussian random vector with $N(0,1)$ independent components, we have the stochastic representation (see [13])

$$
\left(X_{1}(0), \ldots, X_{m}(0), X_{m+1}(0)\right) \stackrel{d}{=} R\left(\boldsymbol{O B}, I \sqrt{1-B^{2}}\right),
$$

where $\boldsymbol{O}=\left(O_{1}, \ldots, O_{m}\right)$ is a random vector uniformly distributed on the unit sphere of $\mathbb{R}^{m}$. Here the rv $I$ satisfies $\mathbb{P}(I= \pm 1)=1 / 2$, the random radius $R>0$ a.s. is such that $R^{2}$ has chi-square distribution with $m+1$ degrees of freedom, and the rv $B$ is supported in $(0,1)$ a.s. such that $B^{2}$ has beta distribution with parameters $m / 2,1 / 2$. Moreover, $\boldsymbol{O}, I, R, B, Z_{i}, i \leq m+1$ are mutually independent. Consequently, using the fact that $\sqrt{x_{0}+x}=\sqrt{x_{0}}+$ $\left(2 \sqrt{x_{0}}\right)^{-1} x(1+o(1))$ as $x \rightarrow 0$, together with (3.25) and (3.26), we obtain as $u \rightarrow \infty$

$$
\begin{aligned}
A_{u}= & \frac{\sum_{i=1}^{m} \rho\left(q t_{j}\right) X_{i}(0)\left[u \Delta_{i}\left(t_{j}\right)\right]-\sum_{i=1}^{m} \frac{u\left(1-\rho^{2}\left(q t_{j}\right)\right)}{2} X_{i}^{2}(0)+\sum_{i=1}^{m} \frac{u \Delta_{i}^{2}\left(t_{j}\right)}{2}}{|\boldsymbol{X}(0)|} \\
& \times\left(1+o_{p}(1)\right) \mid\left(\zeta(0)=u_{x}\right) \\
= & \frac{\sum_{i=1}^{m} \sqrt{2} X_{i}(0) Z_{i}\left(t_{j}\right)-\frac{\sum_{i=1}^{m} X_{i}^{2}(0)}{u} t_{j}^{\alpha}+\frac{\sum_{i=1}^{m} Z_{i}^{2}\left(t_{j}\right)}{u}}{|\boldsymbol{X}(0)|}\left(1+o_{p}(1)\right) \mid\left(\zeta(0)=u_{x}\right) \\
\stackrel{d}{=} & \left(\sqrt{2} \sum_{i=1}^{m} O_{i} Z_{i}\left(t_{j}\right)-\frac{R B}{u} t_{j}^{\alpha}+\frac{\sum_{i=1}^{m} Z_{i}^{2}\left(t_{j}\right)}{u R B}\right) \\
& \times\left(1+o_{p}(1)\right) \mid\left(R\left(\delta B+\sqrt{1-\delta^{2}} \sqrt{1-B^{2}} I\right)=u_{x}\right) \\
B_{u} \stackrel{d}{=} & \left(\sqrt{2} Z_{m+1}\left(t_{j}\right)-\frac{R \sqrt{1-B^{2}} I}{u} t_{j}^{\alpha}\right)\left(1+o_{p}(1)\right) \mid\left(\zeta(0)=u_{x}\right) .
\end{aligned}
$$

Since the following stochastic representation

$$
\sum_{i=1}^{m} O_{i} Z_{i}\left(t_{j}\right) \stackrel{d}{=} Z_{1}\left(t_{j}\right)\left(\sum_{i=1}^{m} O_{i}^{2}\right)^{1 / 2}=Z_{1}\left(t_{j}\right)
$$

holds, we have further by 3.24

$$
\begin{aligned}
& \delta A_{u}+\sqrt{1-\delta^{2}} B_{u} \\
& \stackrel{d}{=}\left(\sqrt{2}\left(\delta \sum_{i=1}^{m} O_{i} Z_{i}\left(t_{j}\right)+\sqrt{1-\delta^{2}} Z_{m+1}\left(t_{j}\right)\right)-\frac{R\left(\delta B+\sqrt{1-\delta^{2}} \sqrt{1-B^{2}} I\right)}{u} t_{j}^{\alpha}\right. \\
& \left.\quad+\frac{\sum_{i=1}^{m} Z_{i}^{2}\left(t_{j}\right)}{u R B}\right)\left(1+o_{p}(1)\right) \mid\left(R\left(\delta B+\sqrt{1-\delta^{2}} \sqrt{1-B^{2}} I\right)=u_{x}\right) \\
& \stackrel{d}{\rightarrow} \sqrt{2} Z\left(t_{j}\right)-t_{j}^{\alpha}, \quad u \rightarrow \infty
\end{aligned}
$$

establishing the convergence for any fixed $t_{j}>0$. The joint convergence in distribution for $0<t_{1}<\cdots<t_{d}<\infty$ can be shown with similar arguments and is therefore omitted here. 
Lemma 7 Under the assumptions and the notation of Lemma 6 , for any a, $T$ positive

$$
\limsup _{u \rightarrow \infty} \sum_{j=N}^{[T /(a q)]} \mathbb{P}(\zeta(a q j)>u \mid \zeta(0)>u) \rightarrow 0, \quad N \rightarrow \infty .
$$

Proof. It follows from (2.9) that for any $\varepsilon>0$ small enough

$$
\frac{1}{2} t^{\alpha} \leq 1-\rho(t) \leq 2 t^{\alpha}, \quad \forall t \in(0, \epsilon]
$$

Denote by $\boldsymbol{X}(q t)-\rho(q t) \boldsymbol{X}(0)=\left(X_{1}(q t)-\rho(t) X_{1}(0), \ldots, X_{m}(q t)-\rho(q t) X_{m}(0)\right)$, and define

$$
\zeta^{*}(q t) \equiv \delta|\boldsymbol{X}(q t)-\rho(q t) \boldsymbol{X}(0)|+\sqrt{1-\delta^{2}}\left(X_{m+1}(q t)-\rho(q t) X_{m+1}(0)\right) .(3.27)
$$

Since $X(q t)-\rho(q t) X(0)$ is independent of $X(0)$, and $X(q t)-\rho(q t) X(0) \stackrel{d}{=}$ $\sqrt{1-\rho^{2}(q t)} X(0)$, we have that $\zeta^{*}(q t)$ is independent of $\zeta(0)$, and

$$
\zeta^{*}(q t) \stackrel{d}{=} \sqrt{1-\rho^{2}(q t)} \zeta(0) .
$$

Moreover, by the triangle inequality

$$
\begin{aligned}
\zeta^{*}(q t) & \geq\left(\delta|\boldsymbol{X}(q t)|+\sqrt{1-\delta^{2}} X_{m+1}(q t)\right)-\rho(q t)\left(\delta|\boldsymbol{X}(0)|+\sqrt{1-\delta^{2}} X_{m+1}(0)\right) \\
& =\zeta(q t)-\rho(q t) \zeta(0)>u(1-\rho(q t))
\end{aligned}
$$

provided that $\zeta(q t)>\zeta(0)>u$. Therefore

$$
\begin{aligned}
\mathbb{P}(\zeta(q t)>u \mid \zeta(0)>u) & \leq 2 \frac{\mathbb{P}(\zeta(q t)>\zeta(0)>u)}{\mathbb{P}(\zeta(0)>u)} \\
& \leq 2 \frac{\mathbb{P}\left(\zeta^{*}(q t)>u(1-\rho(q t)), \zeta(0)>u\right)}{\mathbb{P}(\zeta(0)>u)} \\
& =2 \mathbb{P}\left(\zeta(0)>u \sqrt{\frac{1-\rho(q t)}{1+\rho(q t)}}\right) .
\end{aligned}
$$

Furthermore, it follows from Chebyshev's inequality and Lemma 5 that for any $p>m$

$$
\begin{aligned}
\mathbb{P}(\zeta(q t)>u \mid \zeta(0)>u) & \leq\left\{\begin{array}{l}
2^{1+p \frac{\mathbb{E}\left(|\zeta(0)|^{p}\right)}{t^{\alpha p / 2}},} q t \in(0, \epsilon] \\
2 \mathbb{P}\left(\zeta(0)>u \sqrt{\frac{\lambda}{2}}\right), q t \in(\epsilon, T]
\end{array}\right. \\
& \leq\left\{\begin{array}{l}
K_{p} t^{-\alpha p / 2}, q t \in(0, \epsilon] \\
K_{p} u^{m-1-p}, q t \in(\epsilon, T]
\end{array}\right.
\end{aligned}
$$

is satisfied for some positive constant $K_{p}$, where $\lambda=1-\sup _{\epsilon<s \leq T} \rho(s)>0$, and the second inequality is due to the fact that

$$
\mathbb{P}(\zeta(0)>u) \leq C u^{m-1} \frac{1}{\sqrt{2 \pi} u} \exp \left(-\frac{u^{2}}{2}\right) \leq C_{p} u^{m-1-p}, \quad u>0
$$


holds for some positive constants $C$ and $C_{p}$. Hence, if $p=2(2 / \alpha+m-1)$, then for $t \geq 1$

$$
\begin{aligned}
\mathbb{P}(\zeta(q t)>u \mid \zeta(0)>u) & \leq K_{p}\left(1+T^{\alpha(p-m+1) / 2}\right) \max \left(t^{-\alpha p / 2}, t^{-\alpha(p-m+1) / 2}\right) \\
& \leq C_{p} t^{-2}, \quad q t \in(0, T] .
\end{aligned}
$$

Consequently,

$$
\limsup _{u \rightarrow \infty} \sum_{j=N}^{[T /(a q)]} \mathbb{P}(\zeta(a q j)>u \mid \zeta(0)>u) \leq C_{p} \sum_{j=N}^{\infty}(a j)^{-2} \rightarrow 0, \quad N \rightarrow \infty
$$

establishing the proof.

Lemma 8 Under the assumptions and the notation of Lemma 6 there exist positive constants $C, p, \lambda_{0}, u_{0}$ and $d>1$ such that

$$
\mathbb{P}\left(\zeta(q t)>u+\frac{\lambda}{u}, \zeta(0) \leq u\right) \leq C t^{d} \lambda^{-p} \mathbb{P}(\zeta(0)>u)
$$

for any positive $t$ satisfying $0<t^{\alpha / 2}<\lambda<\lambda_{0}$ and all $u>u_{0}$.

Proof. By (2.9) there exists $\epsilon>0$ such that

$$
\rho(t) \geq \frac{1}{2} \quad \text { and } \quad 1-\rho(t) \leq 2 t^{\alpha}
$$

for all $t \in(0, \epsilon]$. Further, for any $t$ positive satisfying $0<t^{\alpha / 2}<\lambda<\lambda_{0}:=$ $\min \left(1 / 8, \epsilon^{\alpha / 2}\right)$ and $u>1$

$$
\frac{1}{\rho(q t)}-1 \leq 4 \frac{t^{\alpha}}{u^{2}} \leq \frac{\lambda}{2 u^{2}}
$$

Next, for

$$
\boldsymbol{X}_{1 / \rho}(q t)=\left(X_{1}(q t)-\rho^{-1}(q t) X_{1}(0), \ldots, X_{m}(q t)-\rho^{-1}(q t) X_{m}(0)\right)
$$

we have by the triangle inequality

$$
|\boldsymbol{X}(q t)| \leq\left|\boldsymbol{X}_{1 / \rho}(q t)\right|+\frac{1}{\rho(q t)}|\boldsymbol{X}(0)| .
$$

Further, letting

$$
\zeta^{* *}(q t)=\delta\left|\boldsymbol{X}_{1 / \rho}(q t)\right|+\sqrt{1-\delta^{2}}\left(X_{m+1}(q t)-\rho^{-1}(q t) X_{m+1}(0)\right),
$$


and by utilising similar arguments as for $\zeta^{*}$ given in (3.27), we have that $\zeta^{* *}(q t)$ is independent of $\zeta(q t)$ and $\zeta^{* *}(q t) \stackrel{d}{=} \sqrt{1-\rho^{2}(q t)} / \rho(q t) \zeta(0)$. Therefore, for any $t$ positive satisfying $0<t^{\alpha / 2}<\lambda<\lambda_{0}$ and $u>1$

$$
\begin{aligned}
& \mathbb{P}\left(\zeta(q t)>u+\frac{\lambda}{u}, \zeta(0) \leq u \mid \zeta(q t)>u\right) \\
& \leq \mathbb{P}\left(\zeta^{* *}(q t)>\frac{\lambda}{u}+u-\frac{\zeta(0)}{\rho(q t)}, \zeta(0) \leq u \mid \zeta(q t)>u\right) \\
& \leq \mathbb{P}\left(\zeta^{* *}(q t)>\frac{\lambda}{u}-\left(\frac{1}{\rho(q t)}-1\right) u \mid \zeta(q t)>u\right) \\
& \leq \mathbb{P}\left(\zeta^{* *}(q t)>\frac{\lambda}{2 u}\right)=\mathbb{P}\left(\zeta(0)>\frac{\rho(q t)}{\sqrt{1-\rho^{2}(q t)}} \frac{\lambda}{2 u}\right) \\
& \leq \mathbb{P}\left(\zeta(0)>\frac{\lambda}{8 t^{\alpha / 2}}\right) .
\end{aligned}
$$

Consequently, by Chebyshev's inequality for any positive constant $p>2 / \alpha$

$$
\mathbb{P}\left(\zeta(q t)>u+\frac{\lambda}{u}, \zeta(0) \leq u\right) \leq 8^{p} \mathbb{E}\left(|\zeta(0)|^{p}\right) t^{\alpha p / 2} \lambda^{-p} \mathbb{P}(\zeta(q t)>u)
$$

holds for any $t$ positive satisfying $0<t^{\alpha / 2}<\lambda<\lambda_{0}$ and $u$ large. Thus the proof is complete.

Proof of Theorem 2.2 With Lemma 5 Lemma 8 we conclude that the claim follows by an application of Theorem 1.1

\subsection{Proof of Theorem 2.3}

In view of 3,6 or 21, we need to verify two additional conditions (see Lemmas 9 and 10) for the order statistics processes generated by the stationary Gaussian process $X$.

Lemma 9 Under the assumptions of Theorem 2.3, we have for any constants $a, T>0$

$$
\lim _{\varepsilon \downarrow 0} \limsup _{u \rightarrow \infty} \sum_{j=[T /(a q)]}^{\left[\varepsilon / \mathbb{P}\left(X_{n: n}(0)>u\right)\right]} \mathbb{P}\left(X_{n: n}(a q j)>u \mid X_{n: n}(0)>u\right)=0 .
$$


Proof. Recalling that $X(t)-\rho(t) X(0)$ is independent of $X(0)$,

$$
\begin{aligned}
& \mathbb{P}\left(X_{n: n}(t)>u \mid X_{n: n}(0)>u\right)=\mathbb{P}\left(X_{n: n}(t)>u, X_{n: n}(0)>u \mid X_{n: n}(0)>u\right) \\
& =2^{n}(\mathbb{P}(X(t)>X(0)>u \mid X(0)>u))^{n} \\
& \leq 2^{n}(\mathbb{P}(X(t)-\rho(t) X(0)>u(1-\rho(t)), X(0)>u \mid X(0)>u))^{n} \\
& \leq 2^{n}\left(1-\Phi\left(u \sqrt{\frac{1-|\rho(t)|}{1+|\rho(t)|}}\right)\right)^{n} \\
& \leq K u^{-n}\left(\frac{1-|\rho(t)|}{1+|\rho(t)|}\right)^{-n / 2} \exp \left(-\frac{n u^{2}}{2} \frac{1-|\rho(t)|}{1+|\rho(t)|}\right)
\end{aligned}
$$

holds for some positive constant $K$ and $u$ large (the constant $K$ below may be different from line to line), here $\Phi(\cdot)$ denotes the standard normal df.

Now we choose a function $g=g(u)$ such that $\lim _{u \rightarrow \infty} g(u)=\infty,|\rho(g(u))|=$ $u^{-2}$. It follows from $u^{-2} \ln g(u)=o(1)$ that $g(u) \leq \exp \left(\epsilon^{\prime} u^{2}\right)$ for some $0<$ $\epsilon^{\prime}<n / 2(1-|\rho(T)|) /(1+|\rho(T)|)$ and sufficiently large $u$. Now we split the sum in (3.29) at $a q j=g(u)$. The first term satisfies

$$
\begin{aligned}
& \sum_{j=[T /(a q)]}^{[g(u) /(a q)]} \mathbb{P}\left(X_{n: n}(a q j)>u \mid X_{n: n}(0)>u\right) \\
& \quad \leq K \frac{g(u)}{a q} u^{-n}\left(\frac{1-|\rho(T)|}{1+|\rho(T)|}\right)^{-n / 2} \exp \left(-\frac{n u^{2}}{2} \frac{1-|\rho(T)|}{1+|\rho(T)|}\right) \\
& \leq K u^{2 / \alpha-n} \exp \left(\epsilon^{\prime} u^{2}-\frac{n u^{2}}{2} \frac{1-|\rho(T)|}{1+|\rho(T)|}\right) \\
& \quad \rightarrow 0, \quad u \rightarrow \infty
\end{aligned}
$$

since $\epsilon^{\prime}<n / 2(1-|\rho(T)|) /(1+|\rho(T)|)$. For the remaining term we have

$$
\begin{aligned}
& \sum_{j=[g(u) /(a q)]}^{\left[\varepsilon / \mathbb{P}\left(X_{n: n}(0)>u\right)\right]} \mathbb{P}\left(X_{n: n}(a q j)>u \mid X_{n: n}(0)>u\right) \\
& \leq K \frac{\varepsilon}{\mathbb{P}\left(X_{n: n}(0)>u\right)} u^{-n}\left(\frac{1-u^{-2}}{1+u^{-2}}\right)^{-n / 2} \exp \left(-\frac{n u^{2}}{2} \frac{1-u^{-2}}{1+u^{-2}}\right) \\
& \leq K \varepsilon \exp \left(-\frac{n u^{2}}{2}\left(\frac{1-u^{-2}}{1+u^{-2}}-1\right)\right) \\
& \leq K \varepsilon, \quad u \rightarrow \infty .
\end{aligned}
$$

Therefore, the claim follows by taking $\varepsilon \downarrow 0$.

In the following lemma we shall establish the asymptotic independence of $X_{n: n}$ over suitable separate intervals (see condition $D^{\prime}$ in 3 ). In the notation used below $\widetilde{\mathcal{A}}_{n, \alpha}$ is the constant appearing in (2.12), and

$$
T=T(u)=\frac{(2 \pi)^{n / 2}}{\widetilde{\mathcal{A}}_{n, \alpha}} u^{n-\frac{2}{\alpha}} \exp \left(\frac{n u^{2}}{2}\right) .
$$


Lemma 10 Under the assumptions of Theorem 2.3, if futher $T=T(u)$ is defined by (3.31) and $a>0,0<\lambda<1$ are given constants, then for any $0 \leq s_{1}<\cdots<s_{p}<t_{1}<\cdots<t_{p^{\prime}}$ in $\{a q j: j \in \mathbb{Z}, 0 \leq a q j \leq T\}$ with $t_{1}-s_{p} \geq \lambda T$ we have

$$
\begin{aligned}
\lim _{u \rightarrow \infty} \mid \mathbb{P}\left(\bigcap_{i=1}^{p}\left\{X_{n: n}\left(s_{i}\right) \leq u\right\}, \bigcap_{j=1}^{p^{\prime}}\left\{X_{n: n}\left(t_{j}\right) \leq u\right\}\right) \\
\quad-\mathbb{P}\left(\bigcap_{i=1}^{p}\left\{X_{n: n}\left(s_{i}\right) \leq u\right\}\right) \mathbb{P}\left(\bigcap_{j=1}^{p^{\prime}}\left\{X_{n: n}\left(t_{j}\right) \leq u\right\}\right) \mid=0 .
\end{aligned}
$$

Proof. First, taking logarithms on both sides of (3.31) we obtain

$$
\ln T=\frac{n u^{2}}{2}+\left(n-\frac{2}{\alpha}\right) \ln u+\ln \left(\frac{(2 \pi)^{n / 2}}{\widetilde{\mathcal{A}}_{n, \alpha}}\right)
$$

which together with $u^{2}=(2 / n) \ln T(1+o(1))$ implies that

$$
u^{2}=\frac{2 \ln T}{n}+\left(\frac{2}{n \alpha}-1\right) \ln \ln T+\ln \left(\frac{n}{2}\right)^{1-\frac{2}{n \alpha}} \frac{\left(\widetilde{\mathcal{A}}_{n, \alpha}\right)^{\frac{2}{n}}}{2 \pi}(1+o(1))
$$

as $T \rightarrow \infty$. Further, define (hereafter $\mathbb{I}\{\cdot\}$ denotes the indicator function)

$X_{i j}=X_{j}\left(s_{i}\right) \mathbb{I}\{i \leq p\}+X_{j}\left(t_{i-p}\right) \mathbb{I}\left\{p<i \leq p+p^{\prime}\right\}, \quad 1 \leq i \leq p+p^{\prime}, 1 \leq j \leq n$, and $\left\{Y_{i j}, 1 \leq i \leq p, 1 \leq j \leq n\right\} \stackrel{d}{=}\left\{X_{i j}, 1 \leq i \leq p, 1 \leq j \leq n\right\}$, independent of $\left\{Y_{i j}, p+1 \leq i \leq p+p^{\prime}, 1 \leq j \leq n\right\} \stackrel{d}{=}\left\{X_{i j}, p+1 \leq i \leq p+p^{\prime}, 1 \leq j \leq n\right\}$. Applying Lemma 12 with $X_{i(n)}=X_{n: n}\left(s_{i}\right) \mathbb{I}\{i \leq p\}+X_{n: n}\left(t_{i-p}\right) \mathbb{I}\{p<i \leq$ $\left.p+p^{\prime}\right\}$ (see the Appendix), using similar arguments as in Lemma 8.2.4 in 21] we obtain that the left-hand side of (3.32) is bounded from above by

$$
\begin{aligned}
& K u^{-2(n-1)}\left(\frac{T}{q}\right) \sum_{\lambda T \leq t_{j}-s_{i} \leq T} e^{-\frac{n u^{2}}{1+\left|\rho\left(t_{j}-s_{i}\right)\right|}} \int_{0}^{\left|\rho\left(t_{j}-s_{i}\right)\right|} \frac{(1+|h|)^{2(n-1)}}{\left(1-h^{2}\right)^{n / 2}} d h \\
& \leq K u^{-2(n-1)}\left(\frac{T}{q}\right) \sum_{\lambda T \leq a q j \leq T}|\rho(a q j)| e^{-\frac{n u^{2}}{1+|\rho(a q j)|}}, \quad \text { for large } u,
\end{aligned}
$$

where $K$ is some positive constant. The rest of the proof consists of the same arguments as that of Lemma 12.3.1 in [21] by using (3.33) and the Berman's condition $\rho(t) \ln t=o(1)$. Hence the proof is complete.

Proof of Theorem 2.3 Since Theorem 2.2 and Lemmas 9 and 10 hold for the $n$th order statistics process $X_{n: n}$, in view of Lemma B in [6] we have for $T=T(u)$ defined as in (3.31)

$$
\lim _{u \rightarrow \infty} \mathbb{P}\left(\sup _{t \in[0, T(u)]} X_{n: n}(t) \leq u+\frac{x}{n u}\right)=\exp \left(-e^{-x}\right), \quad x \in \mathbb{R} .
$$

Hence the proof follows by expressing $u$ in terms of $T$ as in (3.33). 


\section{Appendix}

Let $\boldsymbol{X}=\left(X_{1}, \ldots, X_{d}\right)$ and $\boldsymbol{Y}=\left(Y_{1}, \ldots, Y_{d}\right)$ be two Gaussian random vectors with $N(0,1)$ components and covariance matrices $\Sigma^{1}=\left(\sigma_{i j}^{(1)}\right)$ and $\Sigma^{0}=$ $\left(\sigma_{i j}^{(0)}\right)$, respectively. The most elaborated version of Berman's inequality is due to Li and Shao 22], where it is shown that for $\boldsymbol{u}=\left(u_{1}, \ldots, u_{d}\right) \in \mathbb{R}^{d}$ (hereafter the notation $\boldsymbol{x} \leq \boldsymbol{y}$ for any $\boldsymbol{x}, \boldsymbol{y} \in \mathbb{R}^{d}$ means $x_{i} \leq y_{i}$ for all $\left.i \leq d\right)$

$$
|\mathbb{P}(\boldsymbol{X} \leq \boldsymbol{u})-\mathbb{P}(\boldsymbol{Y} \leq \boldsymbol{u})| \leq \frac{1}{2 \pi} \sum_{1 \leq i<j \leq d} A_{i j} \exp \left(-\frac{u_{i}^{2}+u_{j}^{2}}{2\left(1+\rho_{i j}\right)}\right),
$$

where $\rho_{i j}:=\max \left(\left|\sigma_{i j}^{(1)}\right|,\left|\sigma_{i j}^{(0)}\right|\right), A_{i j}:=\left|\arcsin \left(\sigma_{i j}^{(1)}\right)-\arcsin \left(\sigma_{i j}^{(0)}\right)\right|$.

Our goal is to establish $\mathrm{Li}$ and Shao's extension of comparison lemma (Berman's inequality) for the minimum and the maximum order statistics of Gaussian random vectors. Let therefore $\boldsymbol{X}_{j}=\left(X_{i j}, i \leq d\right), j \leq n$ be $n$ independent copies of $\boldsymbol{X}$. Denote the minimum and maximum order statistics vector $\boldsymbol{X}_{n: n}=\left(X_{i(n)}, i \leq d\right), \boldsymbol{X}_{1: n}=\left(X_{i(1)}, i \leq d\right)$ with $X_{i(n)}=\min _{j \leq n} X_{i j}, X_{i(1)}=$ $\max _{j \leq n} X_{i j}, i \leq d$. Similarly, for $\boldsymbol{Y}_{j}, j \leq n$ independent copies of $\boldsymbol{Y}$ we define the minimum and maximum order statistics vectors $\boldsymbol{Y}_{n: n}$ and $\boldsymbol{Y}_{1: n}$, respectively.

Lemma 11 For arbitrary $\boldsymbol{u} \in \mathbb{R}^{d}$ and $k=1, n$ we have

$$
\left|\mathbb{P}\left(\boldsymbol{X}_{k: n} \leq \boldsymbol{u}\right)-\mathbb{P}\left(\boldsymbol{Y}_{k: n} \leq \boldsymbol{u}\right)\right| \leq \frac{n}{2 \pi} \sum_{1 \leq i<j \leq d} A_{i j} \exp \left(-\frac{u_{i}^{2}+u_{j}^{2}}{2\left(1+\rho_{i j}\right)}\right)
$$

Proof. Note that $-\boldsymbol{X}$ and $-\boldsymbol{Y}$ have the same distributions as those of $\boldsymbol{X}$ and $\boldsymbol{Y}$, respectively. Using Theorem 2.1 in [23] with constants $\lambda_{i j}=-u_{i}, i \leq$ $d, j \leq n$, we have

$$
\begin{aligned}
& \left|\mathbb{P}\left(\boldsymbol{X}_{n: n} \leq \boldsymbol{u}\right)-\mathbb{P}\left(\boldsymbol{Y}_{n: n} \leq \boldsymbol{u}\right)\right| \\
& \quad=\left|\mathbb{P}\left(\cup_{i=1}^{d} \cap_{j=1}^{n}\left\{-Y_{i j} \leq-u_{i}\right\}\right)-\mathbb{P}\left(\cup_{i=1}^{d} \cap_{j=1}^{n}\left\{-X_{i j} \leq-u_{i}\right\}\right)\right| \\
& \quad \leq \frac{n}{2 \pi} \sum_{1 \leq i<l \leq d} A_{i l} \exp \left(-\frac{u_{i}^{2}+u_{l}^{2}}{2\left(1+\rho_{i l}\right)}\right) .
\end{aligned}
$$

Next, since $\left|a^{n}-b^{n}\right| \leq n|a-b|, a, b \in[0,1]$ and $n \in \mathbb{N}$, we have by (4.34)

$$
\begin{aligned}
\left|\mathbb{P}\left(\boldsymbol{X}_{1: n} \leq \boldsymbol{u}\right)-\mathbb{P}\left(\boldsymbol{Y}_{1: n} \leq \boldsymbol{u}\right)\right| & =\left|(\mathbb{P}(\boldsymbol{X} \leq \boldsymbol{u}))^{n}-(\mathbb{P}(\boldsymbol{Y} \leq \boldsymbol{u}))^{n}\right| \\
& \leq \frac{n}{2 \pi} \sum_{1 \leq i<l \leq d} A_{i l} \exp \left(-\frac{u_{i}^{2}+u_{l}^{2}}{2\left(1+\rho_{i l}\right)}\right),
\end{aligned}
$$

hence (4.35) for $k=1$ follows and thus the proof is complete. 
Lemma 12 Let $\boldsymbol{X}_{n: n}$ and $\boldsymbol{Y}_{n: n}$ be the minimum order statistics vectors defined above. Then, for all $\boldsymbol{u}>\mathbf{0}$

$\left|\mathbb{P}\left(\boldsymbol{X}_{n: n} \leq \boldsymbol{u}\right)-\mathbb{P}\left(\boldsymbol{Y}_{n: n} \leq \boldsymbol{u}\right)\right| \leq \frac{n}{(2 \pi)^{n}} u^{-2(n-1)} \sum_{1 \leq i<l \leq d}\left|A_{i l}^{*}\right| \exp \left(-\frac{n u^{2}}{1+\rho_{i l}}\right)$,

where $u=\min _{1 \leq i \leq n} u_{i}$ and

$$
A_{i l}^{*}=\int_{\sigma_{i l}^{(0)}}^{\sigma_{i l}^{(1)}} \frac{(1+|h|)^{2(n-1)}}{\left(1-h^{2}\right)^{n / 2}} d h .
$$

Proof. We follow the idea of the proof of Theorem 2.1 in [23]. Let $\left\{Z_{i j}^{h}, i \leq\right.$ $d, j \leq n\}$ be $N(0,1)$ rvs with covariance matrix $\Sigma^{h}=\left(\sigma_{i j, l k}^{h}\right)$ where

$$
\sigma_{i j, l k}^{h}=\mathbb{E}\left(Z_{i j}^{h} Z_{l k}^{h}\right)=\tau_{i l}^{h} \mathbb{I}\{j=k\}, \quad i, l \leq d, j, k \leq n, h \in[0,1]
$$

with $\tau_{i l}^{h}:=h \sigma_{i l}^{(1)}+(1-h) \sigma_{i l}^{(0)}$. Clearly, $Z_{j}^{h}=\left\{Z_{i j}^{h}, i \leq d\right\}, j \leq n$ are independent and identically $N_{d}\left(0, \Sigma^{h}\right)$ distributed with $\Sigma^{h}=h \Sigma^{1}+(1-h) \Sigma^{0}$. Without loss of generality, we assume that $\Sigma^{1}$ and $\Sigma^{0}$ are positive definite. Consequently, we have (see (3.4) and (3.19) in [23])

$$
\begin{aligned}
& \mathbb{P}\left(\boldsymbol{X}_{n: n} \leq \boldsymbol{u}\right)-\mathbb{P}\left(\boldsymbol{Y}_{n: n} \leq \boldsymbol{u}\right) \\
& =\mathbb{P}\left(\boldsymbol{Z}_{1: n}^{1} \geq-\boldsymbol{u}\right)-\mathbb{P}\left(\boldsymbol{Z}_{1: n}^{0} \geq-\boldsymbol{u}\right) \\
& =n \sum_{1 \leq i<l \leq d}\left(\sigma_{i l}^{(1)}-\sigma_{i l}^{(0)}\right) \int_{0}^{1} d h\left(\varphi\left(-u_{i},-u_{l} ; \tau_{i l}^{h}\right)\right. \\
& \quad \times \mathbb{P}\left(\cap_{s=1, s \neq i, l}^{d}\left\{W_{s}^{h}>-u_{s}\right\} \cap_{t=2}^{n}\left\{Z_{i t}^{h} \leq-u_{i}, Z_{l t}^{h} \leq-u_{l}\right\}\right),
\end{aligned}
$$

where $W_{s}^{h}=\max _{1 \leq j \leq n} Z_{s j}^{h}$, and $\varphi\left(-u_{i},-u_{l} ; \tau_{i l}^{h}\right)$ is the bivariate pdf of $\left(Z_{i 1}^{h}, Z_{l 1}^{h}\right)$ which satisfies

$$
\begin{aligned}
\varphi\left(-u_{i},-u_{l} ; \tau_{i l}^{h}\right) & =\frac{1}{2 \pi\left(1-\left(\tau_{i l}^{h}\right)^{2}\right)^{1 / 2}} \exp \left(-\frac{u_{i}^{2}-2 \tau_{i l}^{h} u_{i} u_{l}+u_{l}^{2}}{2\left(1-\left(\tau_{i l}^{h}\right)^{2}\right)}\right) \\
& \leq \frac{1}{2 \pi\left(1-\left(\tau_{i l}^{h}\right)^{2}\right)^{1 / 2}} \exp \left(-\frac{u^{2}}{1+\rho_{i l}}\right), \quad u=\min _{1 \leq i \leq n} u_{i} .
\end{aligned}
$$

Next, let $\left(Z_{i}, \tilde{Z}_{l}\right)$ be a bivariate standardized normal random vector with correlation $\left|\tau_{i l}^{h}\right|$ and set $u=\min _{1 \leq i \leq n} u_{i}>0$. Slepian's inequality in [25] and Lemma 2.3 in 24] imply

$$
\begin{aligned}
\mathbb{P}\left(Z_{i t}^{h} \leq-u_{i}, Z_{l t}^{h} \leq-u_{l}\right) & \leq \mathbb{P}\left(Z_{i} \leq-u_{i}, \tilde{Z}_{l} \leq-u_{l}\right) \\
& \leq \frac{\left(1+\left|\tau_{i l}^{h}\right|\right)^{2}}{u^{2}} \varphi\left(u, u ;\left|\tau_{i l}^{h}\right|\right), \quad t \leq n
\end{aligned}
$$


Consequently, with $A_{i l}^{*}$ defined by (4.36) and $x_{+}=\max (x, 0)$

$$
\begin{aligned}
& \mathbb{P}\left(\boldsymbol{X}_{n: n} \leq \boldsymbol{u}\right)-\mathbb{P}\left(\boldsymbol{Y}_{n: n} \leq \boldsymbol{u}\right) \\
& \leq \frac{n}{(2 \pi)^{n}} u^{-2(n-1)} \sum_{1 \leq i<l \leq d}\left(\sigma_{i l}^{(1)}-\sigma_{i l}^{(0)}\right)_{+} \exp \left(-\frac{n u^{2}}{1+\rho_{i l}}\right) \int_{0}^{1} \frac{\left(1+\left|\tau_{i l}^{h}\right|\right)^{2(n-1)}}{\left(1-\left(\tau_{i l}^{h}\right)^{2}\right)^{n / 2}} d h \\
& \leq \frac{n}{(2 \pi)^{n}} u^{-2(n-1)} \sum_{1 \leq i<l \leq d}\left(A_{i l}^{*}\right)_{+} \exp \left(-\frac{n u^{2}}{1+\rho_{i l}}\right),
\end{aligned}
$$

where in the last step we used the equality $\tau_{i l}^{h}=h\left(\sigma_{i l}^{(1)}-\sigma_{i l}^{(0)}\right)+\sigma_{i l}^{(0)}$, and thus

$$
\int_{0}^{1} \frac{\left(1+\left|\tau_{i l}^{h}\right|\right)^{2(n-1)}}{\left(1-\left(\tau_{i l}^{h}\right)^{2}\right)^{n / 2}} d h=\frac{1}{\sigma_{i l}^{(1)}-\sigma_{i l}^{(0)}} \int_{\sigma_{i l}^{(0)}}^{\sigma_{i l}^{(1)}} \frac{(1+|h|)^{2(n-1)}}{\left(1-h^{2}\right)^{n / 2}} d h .
$$

This completes the proof.

Acknowledgements We are grateful to the referees for their careful reading and numerous suggestions which greatly improved the paper.

\section{References}

1. Al-Rawwash, M., Seif, M.: Measuring the surface roughness using the spatial statistics application. J. Appl. Statist. Sci. 15(2, [2006 on cover]), 205-213 (2007)

2. Albin, J.M.P.: On extremal theory for non differentiable stationary processes. PhD Thesis, University of Lund, Sweden (1987)

3. Albin, J.M.P.: On extremal theory for stationary processes. Ann. Probab. 18(1), 92-128 (1990)

4. Albin, J.M.P.: Extremes and crossings for differentiable stationary processes with application to Gaussian processes in $\mathbf{R}^{m}$ and Hilbert space. Stochastic Process. Appl. 42(1), 119-147 (1992)

5. Albin, J.M.P.: On extremal theory for self-similar processes. Ann. Probab. 26(2), 743$793(1998)$

6. Albin, J.M.P., Jarušková, D.: On a test statistic for linear trend. Extremes 6(3), 247-258 (2003)

7. Alodat, M.: An approximation to cluster size distribution of two Gaussian random fields conjunction with application to FMRI data. J. Statist. Plann. Inference 141(7), 2331-2347 (2011)

8. Alodat, M., Al-Rawwash, M.: Skew-Gaussian random field. J. Comput. Appl. Math. 232(2), 496-504 (2009)

9. Alodat, M., Al-Rawwash, M., Jebrini, M.: Duration distribution of the conjunction of two independent $F$ processes. J. Appl. Probab. 47(1), 179-190 (2010)

10. Aue, A., Horváth, L., Hušková, M.: Extreme value theory for stochastic integrals of Legendre polynomials. J. Multivariate Anal. 100(5), 1029-1043 (2009)

11. Berman, S.: Sojourns and extremes of stationary processes. Ann. Probab. 10(1), 1-46 (1982)

12. Berman, S.: Sojourns and extremes of stochastic processes. Wadsworth \& Brooks/Cole Advanced Books \& Software, Pacific Grove, CA (1992)

13. Cambanis, S., Huang, S., Simons, G.: On the theory of elliptically contoured distributions. J. Multivariate Anal. 11(3), 368-385 (1981)

14. Cheng, D., Xiao, Y.: Geometry and excursion probability of multivariate Gaussian random fields. Manuscript (2013) 
15. Dẹbicki, K., Hashorva, E., Ji, L., Tabiś, K.: On the probability of conjunctions of stationary Gaussian processes. Statist. Probab. Lett. 88, 141-148 (2014)

16. Dieker, A.B., Yakir, B.: On asymptotic constants in the theory of Gaussian processes. Bernoulli 20, 1600-1619 (2014)

17. Falk, M., Hüsler, J., Reiss, R.D.: Laws of small numbers: Extremes and rare events. In: DMV Seminar, vol. 23, p. 3rd edn. Birkhäuser, Basel (2010)

18. Farkas, Y., Hashorva, E.: Tail approximation for reinsurance portfolios of Gaussian-like risks. Scand. Act. J. DOI 10.1080/03461238.2013.825639 (2014)

19. Freitas, A., Hüsler, J., Temido, M.: Limit laws for maxima of a stationary random sequence with random sample size. TEST 21(1), 116-131 (2012)

20. Hashorva, E., Ji, L.: Piterbarg theorems for chi-processes with trend. Extremes DOI 10.1007/s10687-014-0201-1 (2014)

21. Leadbetter, M., Lindgren, G., Rootzén, H.: Extremes and related properties of random sequences and processes, vol. 11. Springer Verlag (1983)

22. Li, W., Shao, Q.M.: A normal comparison inequality and its applications. Probab. Theory Related Fields 122(4), 494-508 (2002)

23. Lu, D., Wang, X.: Some new normal comparison inequalities related to Gordon's inequality. Statist. Probab. Lett. 88, 133-140 (2014)

24. Pickands III, J.: Upcrossing probabilities for stationary Gaussian processes. Trans. Amer. Math. Soc. 145, 51-73 (1969)

25. Piterbarg, V.I.: Asymptotic methods in the theory of Gaussian processes and fields, Translations of Mathematical Monographs, vol. 148. American Mathematical Society, Providence, RI (1996)

26. Seleznjev, O.: Asymptotic behavior of mean uniform norms for sequences of Gaussian processes and fields. Extremes 8(3), 161-169 (2006)

27. Tan, Z., Hashorva, E.: Exact asymptotics and limit theorems for supremum of stationary $\chi$-processes over a random interval. Stochastic Process. Appl. 123(8), 2983-2998 (2013)

28. Tan, Z., Wu, C.: Limit laws for the maxima of stationary chi-processes under random index. TEST in press (2014)

29. Turkman, K.F.: Discrete and continuous time series extremes of stationary processes. Handbook of statistics Vol 30. Time Series Methods and Aplications. Eds. T.S. Rao, S.S. Rao and C.R. Rao. Elsevier, 565-580 (2012)

30. Turkman, K.F., Turkman, M.A.A., Pereira, J.M.: Asymptotic models and inference for extremes of spatio-temporal data. Extremes 13(4), 375-397 (2010)

31. Worsley, K., Friston, K.: A test for a conjunction. Statist. Probab. Lett. 47(2), 135-140 (2000)

32. Yakir, B.: Extremes in Random Fields: A Theory and its Applications, Higher Publication Press, Wiley, New York (2013).

33. Zareifard, H., Jafari Khaledi, M.: Non-Gaussian modeling of spatial data using scale mixing of a unified skew Gaussian process. J. Multivariate Anal. 114, 16-28 (2013) 